\title{
Long-term Follow-up Data from Pivotal Studies of Adjuvant Trastuzumab in Early Breast Cancer
}

\author{
Hartmut Kristeleit ${ }^{1}$ - Marina Parton ${ }^{2}$. Mark Beresford ${ }^{3,4}$ • Iain R. Macpherson ${ }^{5}$ • \\ Rajan Sharma $^{6} \cdot$ Loren Lazarus $^{7} \cdot$ Muireann Kelleher ${ }^{6}$
}

Published online: 16 May 2016

(C) The Author(s) 2016. This article is published with open access at Springerlink.com

\begin{abstract}
The addition of adjuvant trastuzumab therapy for 1 year to standard chemotherapy significantly improved disease-free survival and overall survival versus chemotherapy alone in a number of pivotal early breast cancer studies. Here we review long-term follow-up data on the efficacy, cardiac safety, and general safety of trastuzumab in these pivotal studies. We also evaluate ongoing phase II/III adjuvant trials with newer HER2-targeted agents and the efficacy and safety of the recently developed subcutaneous (SC) formulation of trastuzumab in early breast cancer. Long-term follow-up data confirm the significant survival benefit afforded by the addition of trastuzumab to chemotherapy in patients with HER2positive disease, with an acceptable safety profile. Long-term cardiac safety data suggest that the incidence of cardiac adverse events is maintained at a relatively low level with continued follow-up. At this present time, 1 year of trastuzumab treatment remains the standard of care in HER2-positive early breast cancer. Future adjuvant trastuzumab treatment strategies should focus on reducing cardiotoxicity, particularly in elderly pa-
\end{abstract}

Hartmut Kristeleit

Hartmut.Kristeleit@gstt.nhs.uk

1 Guy's and St Thomas' NHS Foundation Trust, London, UK

2 Royal Marsden NHS Foundation Trust and Kingston NHS Foundation Trust, London, UK

3 Royal United Hospital, Bath, UK

4 Bath University, Bath, UK

5 Beatson West of Scotland Cancer Centre, Glasgow, UK

6 St George's Hospital NHS Trust, London, UK

7 Roche Products Ltd, Welwyn Garden City, UK tients, by identifying potential predictive biomarkers of cardiac dysfunction. Clinicians must also decide whether to omit trastuzumab in women who would achieve little benefit from treatment to avoid cardiotoxicity.

\section{Key Points}

We reviewed long-term efficacy and safety data from a number of pivotal studies that reported significant survival benefits with the addition of 1 year of adjuvant trastuzumab therapy to standard chemotherapy in patients with HER2-positive early breast cancer.

The significant survival benefits seen with trastuzumab were maintained with long-term follow-up, with an acceptable safety profile including a relatively low level of cardiac adverse events.

Reducing cardiotoxicity should be a key factor of future adjuvant trastuzumab treatment strategies, particularly in the elderly and in those who would achieve little benefit from treatment.

\section{Introduction}

Thirty years have passed since the discovery of the neu oncogene and subsequent identification of the human epidermal growth factor receptor 2 (HER2) transmembrane receptor tyrosine kinase that it encodes $[1,2]$. Overexpression of HER2 was quickly recognized to confer poor prognosis in women with metastatic breast cancer [3]. This discovery led rapidly to the development of trastuzumab (Herceptin ${ }^{8}$, Genentech, South San Francisco, CA, USA), a humanized HER2targeting monoclonal antibody, and a clinical trials program ultimately leading to a license for the treatment of metastatic breast cancer overexpressing HER2 [4-6]. 
The success of trastuzumab in the metastatic setting generated much optimism for this agent in the adjuvant setting. Approximately $90 \%$ of breast cancer is diagnosed in the early stages of the disease, and it is estimated that HER2-positive tumors account for 15-20\% of invasive breast cancers [7]. Prior to the development of trastuzumab, women with HER2-positive disease faced a worse prognosis than those with HER2-negative disease, including shorter time to relapse, a higher incidence of metastasis, and reduced survival [8].

Subsequently, a number of early breast cancer studies showed that the addition of adjuvant trastuzumab therapy for 1 year to standard chemotherapy significantly improved disease-free survival (DFS) and overall survival (OS) versus chemotherapy alone, with an acceptable safety profile [9-13]. Here we review long-term follow-up data on the efficacy and safety of trastuzumab in these pivotal studies. We also evaluate ongoing adjuvant trials with newer HER2-targeted agents in early breast cancer, as well as the recently developed subcutaneous (SC) formulation of trastuzumab.

\section{Adjuvant Trastuzumab: Long-term Efficacy Data}

Long-term follow-up data from a number of studies in the early breast cancer setting confirm the significant survival benefit afforded by the addition of trastuzumab to chemotherapy in patients with HER2-positive disease.

\subsection{HERA Study}

The randomized, phase III HERA study (HERceptin Adjuvant) assessed the efficacy of trastuzumab in the adjuvant setting, as well as testing two durations of trastuzumab treatment. The study compared 1 or 2 years of trastuzumab with observation alone in 5102 women with HER2-positive early-stage invasive breast cancer who had completed at least four cycles of neoadjuvant or adjuvant chemotherapy.

In patients receiving trastuzumab for 1 year, 127 DFS events were reported versus 220 in the observation group (hazard ratio [HR] $0.54,95 \%$ confidence interval [CI] $0.43-0.67 ; p<0.0001$ ) after a median follow-up of 1 year, corresponding to an $8.4 \%$ absolute benefit in 2-year DFS rate ( $85.8 \%$ vs $77.4 \%$, respectively) [9]. With further maturity, this DFS advantage was maintained (HR 0.76, $95 \%$ CI 0.67-0.86; $p<0.0001$ ) and resulted in a significant OS benefit (HR 0.76 , $95 \%$ CI $0.65-0.88 ; p=0.0005$ ) after a median follow-up of 8 years and crossover of 884 patients ( $52 \%$ ) from observation to trastuzumab (Fig. 1) [14].

Comparison of the efficacy of trastuzumab in patients receiving 2 years versus 1 year of treatment was assessed in a landmark analysis including 3105 patients who were diseasefree 12 months after randomization. At the clinical cutoff date for this analysis, 367 DFS events were recorded in both the 2-year trastuzumab group and the 1-year trastuzumab group, with no significant between-group DFS difference noted (HR $0.99,95 \%$ CI $0.85-1.14 ; p=0.86$ ) [14]. Thus, 2 years of trastuzumab treatment following adjuvant chemotherapy was not more effective than 1 year of treatment. Ten-year follow-up data from HERA will be published in the coming months.

\subsection{NSABP B-31 and NCCTG N9831 Combined Data}

Two studies of adjuvant chemotherapy with or without trastuzumab in women with surgically removed HER2positive breast cancer were sponsored by The National Cancer Institute. The NSABP B-31 trial (National Surgical Adjuvant Breast and Bowel Project B-31) compared doxorubicin and cyclophosphamide (AC) followed by paclitaxel every 3 weeks (group 1) with AC followed by 1 year of concurrent trastuzumab and 12 weeks of paclitaxel (group 2). The NCCTG N9831 trial (North Central Cancer Treatment Group N9831) compared AC followed by weekly paclitaxel (group A), with AC followed by weekly paclitaxel then 1 year of trastuzumab (group B), and AC followed by 1 year of concurrent trastuzumab and 12 weeks of paclitaxel (group C).

A combined analysis of the two studies after a median follow-up of 2 years compared data from 1679 patients in groups 1 and A (control groups) with data from 1672 patients in groups 2 and $C$ (trastuzumab groups) [10]. The sequential group B of the N9831 study was excluded from this analysis. Trastuzumab combined with adjuvant chemotherapy reduced the risk of a DFS event (HR 0.48, $95 \%$ CI 0.39-0.59; $p<0.0001)$ and OS event versus chemotherapy alone (HR $0.67,95 \%$ CI $0.48-0.93 ; p=0.015$ ) [10]. These survival benefits for the trastuzumab group versus the chemotherapy group were maintained after a median follow-up of 4 years, with a relative reduction in DFS event rate of $48 \%$ (HR 0.52, $95 \% \mathrm{CI}$ $0.45-0.60 ; p<0.001)$ and a relative reduction in death rate of $39 \%$ (HR 0.61, $95 \%$ CI $0.50-0.75 ; p<0.001$ ) [15].

Updated analyses after a median follow-up of 8.4 years, including 2018 patients in the control group and 2028 patients in the trastuzumab group, confirmed a substantial survival benefit with the addition of trastuzumab to adjuvant chemotherapy. There was a $37 \%$ relative improvement in OS versus the chemotherapy group (HR $0.63,95 \%$ CI 0.54-0.73; $p<0.001)$ and an increase in the 10 -year OS rate from $75.2 \%$ to $84.0 \%$ [16]. Patients treated with trastuzumab plus chemotherapy also achieved a $40 \%$ improvement in DFS (HR $0.60,95 \%$ CI $0.53-0.68 ; p<0.001)$ versus chemotherapy alone, and an increase in the 10-year DFS rate from $62.2 \%$ to $73.7 \%$ (Fig. 2). All patient subgroups defined by age, hormone receptor status, and tumor size derived similar 
A

\begin{tabular}{|c|c|c|c|c|}
\hline \multicolumn{2}{|c|}{$\begin{array}{l}\text { Median follow-up } \\
\text { (\% follow-up time after } \\
\text { selective crossover) }\end{array}$} & DFS benefit & HR (95\% Cl) & \multirow{2}{*}{$\begin{array}{l}\text { Number of DFS events: } \\
1 \text { year trastuzumab } \\
\text { vs observation }\end{array}$} \\
\hline $2005(0 \%)$ & 1 year & $\mapsto-1$ & $0.54(0.43-0.67)$ & \\
\hline $2006(4.3 \%)$ & 2 years & $H$ & $0.64(0.54-0.76)$ & 218 vs $321 p<0.0001$ \\
\hline $2008(33.8 \%)$ & 4 years & & $0.76(0.66-0.87)$ & 369 vs $458 p<0.0001$ \\
\hline $2012(48.6 \%)$ & 8 years & & $0.76(0.67-0.86)$ & 471 vs $570 p<0.0001$ \\
\hline
\end{tabular}

B

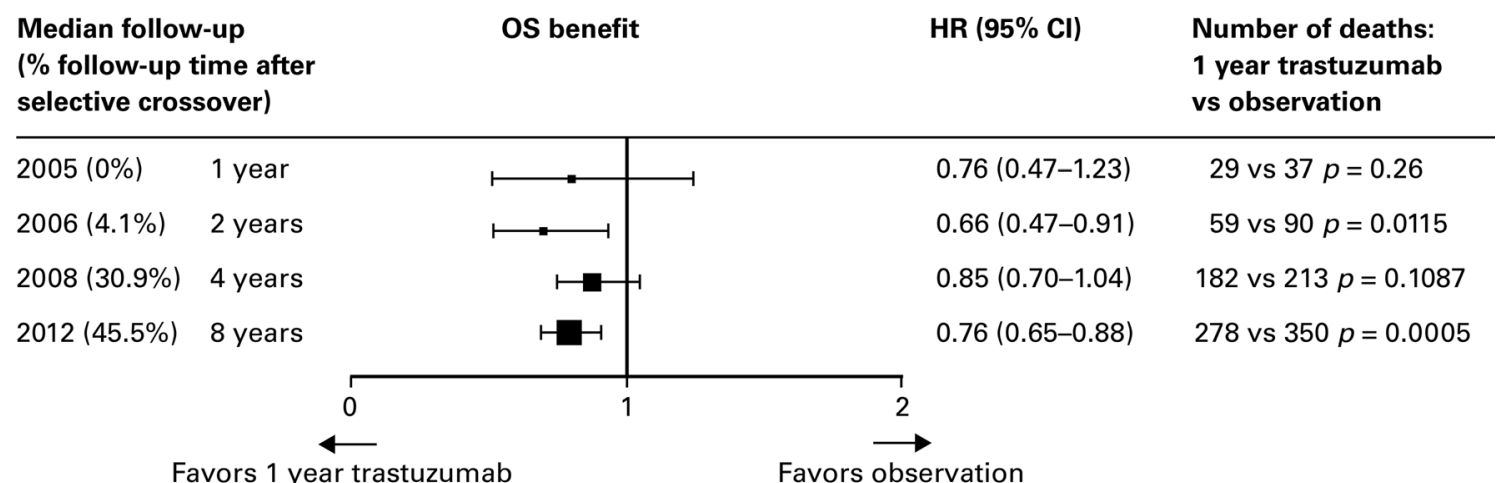

Favors 1 year trastuzumab

Fig. 1 Hazard ratios and confidence intervals for the comparison of a DFS and $\mathbf{b}$ OS with 1 year of trastuzumab versus observation after a median follow-up of 8 years in the HERA study [14]. CI confidence

benefits from the addition of trastuzumab to adjuvant chemotherapy [16].

NCCTG N9831 was the only study to directly compare sequential trastuzumab after completion of anthracyclinebased chemotherapy with delivery concomitant with the taxane portion of chemotherapy. After a median of

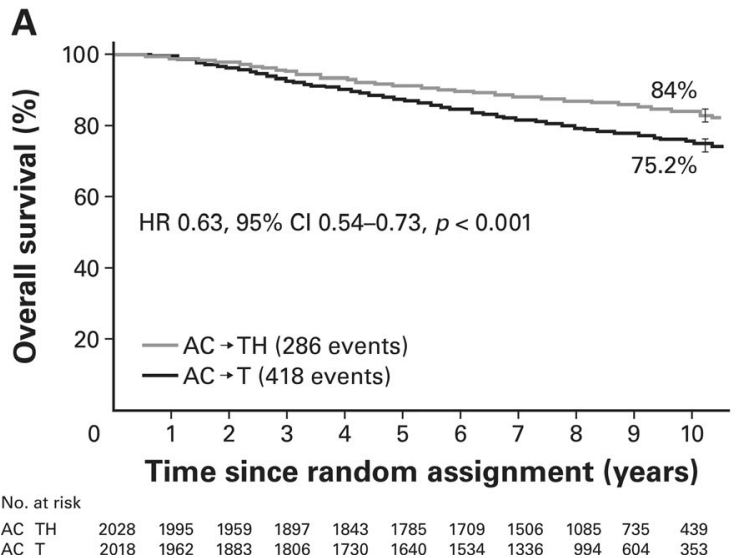

Fig. 2 Combined data analysis of $\mathbf{a}$ OS and $\mathbf{b}$ DFS after a median followup of 8.4 years in the NSABP B-31 and NCCTG N9831 studies [16]. AC $\rightarrow T$ doxorubicin and cyclophosphamide followed by paclitaxel, $A C \rightarrow$

$T H$ doxorubicin and cyclophosphamide followed by paclitaxel and interval, $D F S$ disease-free survival, $H R$ hazard ratio, $O S$ overall survival. Reprinted from [14] with permission from Elsevier

3.9 years of follow-up, a strong trend towards increased 5 -year DFS was noted with concurrent trastuzumab and paclitaxel relative to sequential administration (HR 0.77, $99.9 \%$ CI $0.53-1.11 ; p=0.0216)$, but the $p$ value did not cross the prespecified O'Brien-Fleming boundary for the interim analysis [17].

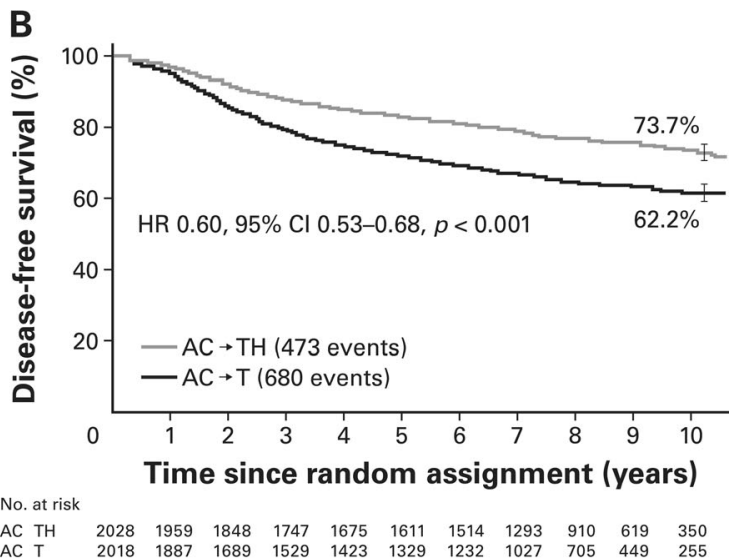

trastuzumab, $C I$ confidence interval, $D F S$ disease-free survival, $H R$ hazard ratio, $O S$ overall survival. Reprinted from [16] with permission from the American Society of Clinical Oncology. All rights reserved 


\subsection{BCIRG 006}

The efficacy and safety of trastuzumab in combination with a non-anthracycline-containing adjuvant chemotherapy regimen was investigated in the BCIRG 006 study (Breast Cancer International Research Group 006). A total of 3222 women with HER2-positive early breast cancer were randomized to receive AC followed by docetaxel every 3 weeks (AC-T), AC-T plus 1 year of trastuzumab, or docetaxel and carboplatin plus 1 year of trastuzumab $(\mathrm{TCH})$.

After a median follow-up of 5.4 years, a significant DFS and OS benefit was observed in both of the trastuzumab-containing groups compared with the AC-T group. Five-year DFS rates were $75 \%$ with AC-T, $84 \%$ with AC-T plus trastuzumab (HR vs AC-T $0.64 ; p<0.001$ ), and $81 \%$ with TCH (HR vs AC-T $0.75 ; p=0.04$ ); 5-year OS rates were $87 \%, 92 \%$ (HR vs AC-T $0.63 ; p<0.001$ ), and $91 \%$ (HR vs AC-T 0.77; $p=0.04$ ), respectively [12]. No significant survival differences were found between the two trastuzumab-containing regimens; however, the study was not powered to detect equivalences between the two groups. Ten-year follow-up data from the study are due to be published in the coming months.

\subsection{NOAH}

The phase III NOAH study (NeOAdjuvant Herceptin) examined the benefit of trastuzumab in the neoadjuvant setting, by comparing neoadjuvant chemotherapy (doxorubicin, paclitaxel, cyclophosphamide, methotrexate, and fluorouracil) plus trastuzumab followed by adjuvant trastuzumab given for a total of 1 year with chemotherapy alone in 334 women with HER2positive locally advanced or inflammatory breast cancer.

Primary results, after a median follow-up of 3.2 years, showed a significant $41 \%$ reduction in the risk of recurrence, progression, or death with the addition of trastuzumab (HR 0.59 , $95 \%$ CI $0.38-0.90 ; p=0.013$ ), with 3-year event-free survival (EFS) rates of $71 \%$ in patients randomized to receive trastuzumab plus chemotherapy versus $56 \%$ in those randomized to receive chemotherapy alone [13]. Corresponding 3-year OS rates were $87 \%$ and $79 \%$, respectively (HR $0.62 ; p=0.114)$. The EFS benefit with trastuzumab was demonstrated in all patient subgroups assessed, including those defined by hormone receptor status or nodal status, achievement of pathologic complete response (pCR) in the breast, or the presence or absence of inflammatory disease [13].

The survival benefit from the addition of trastuzumab to neoadjuvant chemotherapy was sustained after a further 2 years of follow-up, with 5-year EFS rates of $58 \%$ in the trastuzumab group and $43 \%$ in the chemotherapy alone group (HR 0.64, $95 \%$ CI 0.44-0.93; $p=0.016$ ), and 5-year OS rates of $74 \%$ and $63 \%$, respectively (HR $0.66,95 \%$ CI $0.43-1.01$; $p=0.055$ ) [18]. Subgroup analyses of EFS at 5 years were similar to those seen in the primary analysis; patients with inflammatory disease or hormone receptor-negative tumors gained most benefit from the addition of trastuzumab to neoadjuvant chemotherapy (Fig. 3) [18].

\subsection{FinHer}

The phase III FinHer study (Finland Herceptin) examined a short duration of trastuzumab therapy and the effect of sequencing of trastuzumab prior to anthracyclines. In total, 1010 women with node-positive or high-risk node-negative breast cancer were randomized to receive three cycles of docetaxel or vinorelbine followed by three cycles of fluorouracil, epirubicin, and cyclophosphamide (FEC). Within this group, 232 patients with HER2-positive disease were further randomized to receive or not receive trastuzumab for 9 weeks with docetaxel or vinorelbine.

Overall 3-year recurrence-free survival (RFS) rates were higher in the docetaxel group than in the vinorelbine group, at $91.3 \%$ and $86.4 \%$, respectively (HR $0.58,95 \%$ CI $0.40-0.85$; $p=0.005$ ). In addition, patients with HER2-positive early breast cancer receiving trastuzumab had greater 3-year RFS rates than those not receiving trastuzumab $(89.3 \%$ vs $77.6 \%$, respectively; HR 0.42, $95 \%$ CI $0.21-0.83 ; p=0.01$ ) [19].

After a further 2 years' follow-up, patients treated with docetaxel had greater distant DFS than those treated with vinorelbine overall (HR 0.66, $95 \%$ CI 0.49-0.91; $p=0.010)$. Patients with HER2-positive disease treated with trastuzumab tended to have better distant DFS than those treated with chemotherapy alone (HR 0.65, $95 \%$ CI $0.38-1.12 ; p=0.12$ ), with 5-year distant DFS rates of $83.3 \%$ and $73.0 \%$, respectively [20]. In exploratory analyses, distant DFS was superior in patients who received docetaxel, trastuzumab, and FEC compared with those who received docetaxel and FEC (HR 0.32, $95 \%$ CI 0.12-0.89; $p=0.029$ ) or vinorelbine, trastuzumab, and FEC (HR 0.31, $95 \%$ CI $0.11-0.83 ; p=0.020)$. The authors concluded that chemotherapy regimens combined with fewer cycles of trastuzumab should be explored in future studies [20].

\subsection{PHARE}

The French open-label noninferiority PHARE study (Protocol for Herceptin as Adjuvant therapy with Reduced Exposure) compared 6 months with 12 months of adjuvant trastuzumab following completion of at least four cycles of adjuvant chemotherapy. Overall, 1690 patients were eligible for analysis in each arm of the study. After 3.5 years of follow-up, 175 DFS events $(10.4 \%)$ were observed in the 12-month group compared with $219(13.0 \%)$ in the 6-month group (2-year DFS HR $1.28,95 \%$ CI $1.05-1.56 ; p=0.29)$. The study failed to demonstrate noninferiority for the investigational arm. At the time of the primary report the study follow-up was insufficient to provide mature OS data [21]. 
A

Subgroup $n(\%) \quad$ Events $n(\%) \quad H R(95 \% \mathrm{Cl})$

5-year event-free survival (\%)

\begin{tabular}{ll}
\cline { 2 - 2 } & $\begin{array}{l}\text { Trastuzumab and } \\
\text { neoadjuvant chemotherapy }\end{array}$ Neoadjuvant \\
chemotherapy alone
\end{tabular}

\begin{tabular}{|c|c|c|c|}
\hline Overall & $235(100)$ & $111(47)$ & $0.64(0.44-0.93)$ \\
\hline \multicolumn{4}{|l|}{ Age (years) } \\
\hline$\leq 50$ & $108(46)$ & $51(47)$ & $0.95(0.55-1.65)$ \\
\hline$>50$ & $127(54)$ & $60(47)$ & $0.46(0.27-0.78)$ \\
\hline \multicolumn{4}{|l|}{ Nodal status } \\
\hline No & $35(15)$ & $17(49)$ & $0.60(0.23-1.60)$ \\
\hline $\mathrm{N} 1 / \mathrm{N} 2$ & $200(85)$ & $94(47)$ & $0.63(0.42-0.95)$ \\
\hline \multicolumn{4}{|l|}{ pCR* } \\
\hline No & $167(71)$ & $94(56)$ & $0.92(0.61-1.49)$ \\
\hline Yes & $68(29)$ & $17(25)$ & $0.29(0.11-0.78)$ \\
\hline \multicolumn{4}{|l|}{ Inflammatory } \\
\hline No & $188(80)$ & $85(45)$ & $0.76(0.49-1.16)$ \\
\hline Yes & $47(20)$ & $26(55)$ & $0.34(0.15-0.80)$ \\
\hline \multicolumn{4}{|l|}{ ER/PgR } \\
\hline Both negative & $151(64)$ & $66(44)$ & $0.58(0.35-0.94)$ \\
\hline Either positive & $84(36)$ & $45(54)$ & $0.74(0.41-1.44)$ \\
\hline
\end{tabular}

$\begin{array}{ll}58 & 43 \\ 52 & 50 \\ 63 & 38 \\ & \\ 60 & 42 \\ 57 & 44 \\ & \\ 38 & 41 \\ 87 & 55 \\ 56 & 49 \\ 64 & 24 \\ 62 & 48 \\ 49 & 36\end{array}$

B

Subgroup $n(\%)$ Events $n(\%) \quad$ HR $(95 \% \mathrm{Cl})$

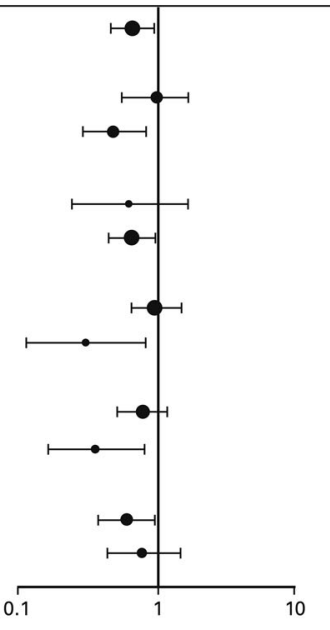

5-year overall survival (\%)

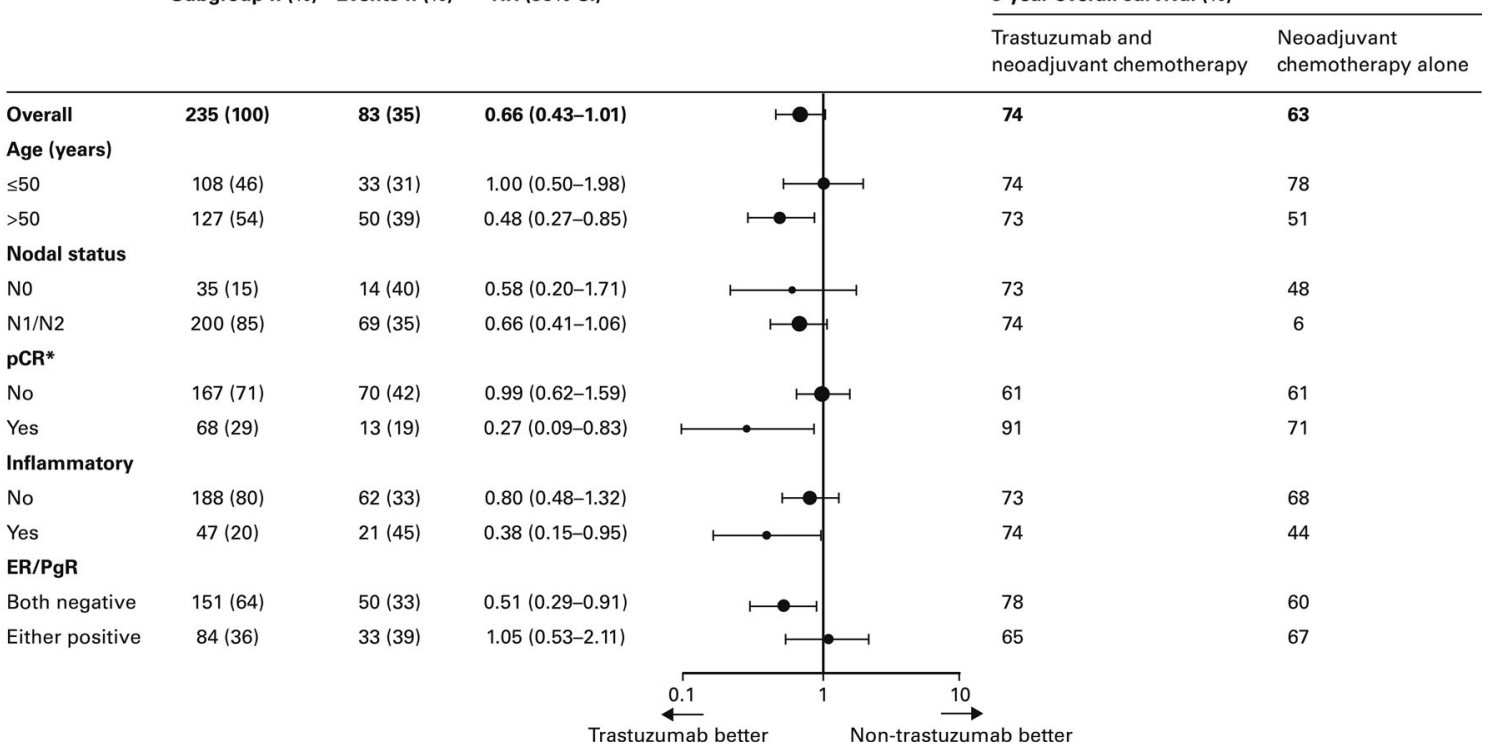

Fig. 3 Subgroup analysis of a EFS and $\mathbf{b}$ OS after a median follow-up of 5.4 years in the NOAH study [18]. CI confidence interval, EFS event-free survival, $E R$ estrogen receptor, $H R$ hazard ratio, $O S$ overall survival, $p C R$

pathologic complete response, $P g R$ progesterone receptor. Reprinted from [18] with permission with permission from Elsevier

\subsection{PACS04}

The efficacy of 1 year of trastuzumab versus observation was examined in 528 patients with axillary node-positive breast cancer, following the completion of adjuvant chemotherapy, as part of a second randomization of the phase III PACS04 (Programme Adjuvant Cancer Sein) trial.

After a median follow-up of 3.9 years, trastuzumab therapy was associated with a non-significant reduction in the risk of relapse versus observation (HR 0.86, $95 \%$ CI 0.61-1.22; $p=0.41$ ); 3-year DFS rates were $81 \%$ and $78 \%$, respectively [22]. There was no significant difference in the risk of death between the trastuzumab arm and the observation arm
(HR 1.27, $95 \%$ CI 0.68-2.38; $p=0.41$ ), with 3-year OS rates of $95 \%$ and $96 \%$, respectively.

\section{Adjuvant Trastuzumab: Long-term Cardiac Safety Findings}

Cardiac dysfunction is a known risk associated with the use of trastuzumab, particularly when trastuzumab is given in combination with anthracyclines [23]. However, unlike anthracycline-related cardiac dysfunction, which is dosedependent and irreversible, trastuzumab-induced cardiotoxicity is independent of dose and mostly reversible 
following treatment discontinuation [24]. Nevertheless, recent reports suggest that long-term cardiac damage may be underestimated and cardiac dysfunction may not return to baseline levels following trastuzumab withdrawal $[25,26]$. Longterm cardiac safety data, reviewed here from the pivotal adjuvant studies of trastuzumab in HER2-positive early breast cancer, suggest that the incidence of cardiac adverse events (AEs) is maintained at a relatively low level with continued follow-up.

\subsection{HERA Study}

Cardiac events occurring at a significantly higher incidence in the 1-year trastuzumab group versus the observation group of the HERA study, after a median follow-up of 1 year, were severe congestive heart failure $(\mathrm{CHF} ; 0.5 \%$ vs $0 \%$, respectively; $p=0.002$ ), symptomatic $\mathrm{CHF}$, including severe $\mathrm{CHF}$ ( $1.7 \%$ vs $0.1 \%$, respectively; $p<0.001)$, and left ventricular ejection fraction (LVEF) decreases $(7.1 \%$ vs $2.2 \%$, respectively; $p<0.001$ ) [9]. One cardiac-related death was recorded in the observation group.

At the 8-year follow-up, cardiac disorders were reported in $1.9 \%$ of patients in both of the trastuzumab treatment groups (Table 1) [14]. Severely symptomatic cardiac events were rare and occurred at a similar frequency in the 1-year and 2-year trastuzumab groups $(0.8 \%$ and $1.0 \%$, respectively). However, asymptomatic or mildly symptomatic cardiac events, including LVEF decreases, were more frequent following 2 years versus 1 year of trastuzumab (7.2\% vs $4.1 \%$, respectively). Cardiac events were rare after completion of trastuzumab therapy [14].

\subsection{NSABP B-31 and NCCTG N9831}

Cardiac safety was reported separately for the NSABP B-31 and NCCTG N9831 studies. The 3-year cumulative incidence of class III or IV CHF or cardiac-related deaths with trastuzumab plus chemotherapy was $4.1 \%$ in NSABP B-31 and $2.9 \%$ in NCCTG N9831 [10]. Cumulative CHF rates after 4 years were $3.8 \%$ and $2.3 \%$, respectively [15]. Rates of cardiac-related death after 8.4 years were $0.2 \%$ with trastuzumab plus chemotherapy and $0.1 \%$ with chemotherapy alone (Table 1) [16].

\subsection{BCIRG 006}

Rates of CHF and relative LVEF reduction of more than $10 \%$ points in the BCIRG 006 study were significantly higher in patients receiving AC-T plus trastuzumab than in those receiving TCH after a median follow-up of 5.4 years $(p<0.001)$ (Table 1) [12]. Of the 194 patients (19\%) in the AC-T plus trastuzumab group with a relative reduction in LVEF of more than $10 \%$, one-third continued to experience LVEF declines at least 4 years after randomization.

\subsection{NOAH}

Cardiac events occurred in $11 \%$ of patients in each of the trastuzumab and no trastuzumab groups at the 3.2-year follow-up [13]. Two patients (1.7\%) experienced asymptomatic LVEF decline and two patients $(1.7 \%)$ developed reversible class III CHF following trastuzumab therapy (Table 1). Safety results were similar at the 5-year follow-up, with four cardiovascular AEs considered to be drug-related (grade 2 lymphostasis and grade 2 lymphedema, each in one patient in the trastuzumab group, and grade 2 thrombosis and grade 2 deep vein thrombosis, each in one patient in the chemotherapy alone group) [18]. The overall incidence of CHF in the trastuzumab group remained at $1.7 \%$.

\subsection{FinHer}

One patient $(0.9 \%)$ in the trastuzumab plus chemotherapy group was diagnosed with symptomatic cardiac failure during the 5 years of follow-up in the FinHer study (Table 1) [20]. The median LVEF of patients treated with trastuzumab and chemotherapy remained at $65 \%$ throughout the 5-year followup period, whereas the median LVEF decreased from $66 \%$ to $62 \%$ in patients receiving chemotherapy alone $(p=0.006)$. A similar proportion of patients in each treatment group experienced a decrease in LVEF of more than $20 \%$ points from baseline [20].

\subsection{PHARE}

The PHARE study observed a significant difference in cardiac events between the 12-month group and the 6-month group (96 [5.7\%] vs 32 [1.9\%] patients, respectively; $p<0.001$ ). The highest proportion of cardiac events was observed in both study arms in patients who had received anthracycline-based chemotherapy without a taxane [21].

\subsection{PACS04}

In total, 29 patients $(11.2 \%)$ in the trastuzumab arm experienced either an LVEF decline of less than $45 \%$ or an LVEF ranging from $45 \%$ to $50 \%$ with a relative decrease of at least $15 \%$ after 3.9 years of follow-up in the PACS04 trial (Table 1) [22]. Symptomatic CHF was reported in four patients $(1.5 \%)$ in the trastuzumab arm and one patient $(0.4 \%)$ in the observation arm. No cardiac-related deaths were recorded.

\section{Cardiac Safety When Adding Other HER2-Targeted Agents}

In the metastatic breast cancer setting, the addition of a second HER2-targeted agent, pertuzumab, to trastuzumab-based 
Table 1 Overview of long-term cardiac safety findings from the pivotal adjuvant studies of trastuzumab

\begin{tabular}{|c|c|c|c|}
\hline \multirow[t]{3}{*}{ Cardiac AEs } & \multicolumn{3}{|l|}{ Number $(\%)$ of patients } \\
\hline & HERA 8 years' follow-up [14] & & \\
\hline & 1 year trastuzumab $(n=1682)$ & 2 years trastuzumab $(n=1673)$ & Observation $(n=1744)$ \\
\hline Grade $3 / 4$ cardiac disorders & $32(1.9)$ & $32(1.9)$ & $8(0.5)$ \\
\hline Severely symptomatic cardiac events* & $14(0.8)$ & $16(1.0)$ & $2(0.1)$ \\
\hline \multirow[t]{3}{*}{ Asymptomatic cardiac events ${ }^{\dagger}$} & $69(4.1)$ & $120(7.2)$ & $15(0.9)$ \\
\hline & \multicolumn{3}{|c|}{ NSABP B-31 and NCCTG N9831 8.4 years' follow-up [16] } \\
\hline & Trastuzumab + chemotherapy $(n=2028)$ & Chemotherapy alone $(n=2018)$ & \\
\hline $\mathrm{CHF}$ & $2(0.1)$ & $3(0.1)$ & \\
\hline Cardiac arrest & $1(<0.1)$ & $3(0.1)$ & \\
\hline Cardiomyopathy & 0 & $1(<0.1)$ & \\
\hline Myocardial infarction & 0 & $1(<0.1)$ & \\
\hline \multirow[t]{3}{*}{ Unspecified cardiac condition } & 0 & $1(<0.1)$ & \\
\hline & \multicolumn{3}{|l|}{ BCIRG 0065.4 years' follow-up [12] } \\
\hline & $\mathrm{AC}-\mathrm{T}(n=1073)$ & AC-T + trastuzumab $(n=1074)$ & $\mathrm{TCH}(n=1075)$ \\
\hline Cardiac-related death & 0 & 0 & 0 \\
\hline Class III/IV CHF & $7(0.7)(n=1018)$ & $21(2.0)(n=1042)$ & $4(0.4)^{*}(n=1031)$ \\
\hline \multirow[t]{3}{*}{ LVEF relative decrease $>10 \%$} & $114(11.2)^{\S}$ & $194(18.6)$ & $97(9.4)^{*}$ \\
\hline & \multicolumn{3}{|l|}{ NOAH 3.2 years' follow-up [13] } \\
\hline & Trastuzumab + chemotherapy $(n=115)$ & Chemotherapy alone $(n=113)$ & \\
\hline Grade 3/4 left ventricular dysfunction & $2(1.7)$ & 0 & \\
\hline LVEF decrease $\geq 20 \%$ points from baseline & $2(1.7)$ & $1(0.9)$ & \\
\hline \multirow[t]{3}{*}{ Class III CHF } & $2(1.7)$ & 0 & \\
\hline & \multicolumn{3}{|l|}{ FinHer 5 years' follow-up [20] } \\
\hline & Trastuzumab + chemotherapy $(n=115)$ & Chemotherapy alone $(n=116)$ & \\
\hline LVEF decrease $>20 \%$ points from baseline & $7(6.8)$ & $10(10.5)$ & \\
\hline Symptomatic heart failure & $1(0.9)$ & $2(1.7)$ & \\
\hline \multirow[t]{3}{*}{ Myocardial infarction } & 0 & 0 & \\
\hline & \multicolumn{3}{|l|}{ PHARE 3.5 years' follow-up [21] } \\
\hline & 12-month group $(n=1690)$ & 6-month group $(n=1690)$ & \\
\hline LVEF $<50 \%$ & $106(6.3)$ & $79(4.7)$ & \\
\hline \multirow[t]{3}{*}{ LVEF $<50 \%$ independent of baseline and a decrease of $>10 \%$} & $81(4.8)$ & $60(3.6)$ & \\
\hline & \multicolumn{3}{|l|}{ PACS04 3.9 years' follow-up [22] } \\
\hline & 1 year trastuzumab $(n=260)$ & Observation $(n=268)$ & \\
\hline Symptomatic CHF & $4(1.5)$ & $1(0.4)$ & \\
\hline LVEF decline $<45$ or $45-50 \%$ with relative decrease $\geq 15 \%$ & $29(11.2)$ & $7(2.6)$ & \\
\hline
\end{tabular}

$A C-T$ doxorubicin and cyclophosphamide followed by docetaxel, $A E$ adverse event, $C H F$ congestive heart failure, $L V E F$ left ventricular ejection fraction, NYHA New York Heart Association, TCH docetaxel and carboplatin plus trastuzumab

*NYHA class III or IV and a significant LVEF drop $\geq 10 \%$ points from baseline and to an absolute LVEF $<50 \%$, or cardiac death

${ }^{\dagger}$ NYHA class I or II with a significant LVEF drop $\geq 10 \%$ points from baseline and to an absolute LVEF $<50 \%$ confirmed by repeat assessment

${ }^{\ddagger} p<0.001$ for the comparison of the AC-T plus trastuzumab group versus the TCH group

${ }^{\S} p<0.001$ for the comparison of the AC-T plus trastuzumab group versus the AC-T group

chemotherapy did not result in any additional cardiac safety concerns in the phase III CLEOPATRA study (CLinical Evaluation Of Pertuzumab And TRAstuzumab) in patients with previously untreated HER2-positive tumors. More patients in the placebo group than in the pertuzumab group experienced grade 3 or higher cardiac AEs (3.8\% vs $1.5 \%$, respectively), as well as serious cardiac AEs (3.3\% vs $1.2 \%$, respectively) [24]. The hope is that this cardiac safety profile will be reproduced in the ongoing phase III
APHINITY trial (Adjuvant Pertuzumab and Herceptin in INItial TherapY of Breast Cancer), which is evaluating the addition of pertuzumab to trastuzumab and chemotherapy in the adjuvant setting (ClinicalTrials.gov: NCT01358877). Long-term cardiac safety data from two pivotal phase II studies of pertuzumab in the neoadjuvant setting, TryPhaena (ToleRabilitY of Pertuzumab, Herceptin and AnthracyclinEs in NeoAdjuvant breast cancer; ClinicalTrials.gov: NCT00976989) and NeoSphere (Neoadjuvant Study of 
Pertuzumab and Herceptin in an Early Regimen Evaluation; Clinical Trials.gov: NCT00545688), are also eagerly awaited.

Despite these encouraging cardiac safety findings, patients receiving trastuzumab or pertuzumab should be routinely monitored for left ventricular function prior to and during treatment to detect early cardiotoxicity. Echocardiograms are widely considered the gold standard monitoring method since they are safe, cost-effective, and readily available, but experienced operators are required as the potential for variability is high. There is some debate as to the optimal LVEF monitoring interval. The US Food and Drug Administration recommends monitoring at baseline and at every 3 months during and upon completion of trastuzumab [27]. The UK National Cancer Research Institute suggests monitoring at baseline and at 4 and 8 months during trastuzumab; further assessment is only recommended if cardiovascular intervention has been required [28]. More recently, the European Society for Medical Oncology clinical practice guidelines suggested cardiac monitoring at baseline, at 3, 6, and 9 months during trastuzumab, and at 12 and 18 months after treatment initiation [29].

\section{Adjuvant Trastuzumab: Long-term Non-cardiac Safety Data}

Table 2 provides an overview of non-cardiac grade 3/4 AEs observed across the pivotal adjuvant studies. Overall, there were few differences in toxicities recorded with the exception of the BCIRG 006 study, in which there was an increased rate of acute leukemia in the anthracycline-containing arms versus the non-anthracycline arm [12]. Seven patients in the anthracycline-containing groups developed acute leukemia, as well as one patient in the TCH group who received an anthracycline outside of the study for lymphoma. Five of these patients died as a result of their leukemia.

Much of the non-cardiac toxicity described in the adjuvant studies was attributable to the chemotherapy backbone rather than the trastuzumab antibody. Allergic reaction was reported in $5.8 \%$ of patients treated with docetaxel plus trastuzumab in the FinHer study, but in $0 \%$ of patients treated with vinorelbine plus trastuzumab [19]. Diarrhea was reported more frequently in the chemotherapy-alone arm of the NOAH study than in the trastuzumab plus chemotherapy arm $(3.5 \%$ vs $0.9 \%$, respectively) [13]. Data on the effects of long-term trastuzumab use and weight gain or dermatologic conditions were scarce in these pivotal trials.

\section{Future Outlook}

Data from a number of ongoing or planned randomized phase II/III trials will help to elucidate the role of newer HER2- targeted agents in early breast cancer. The phase II ATEMPT trial is comparing the antibody-drug conjugate trastuzumab emtansine with paclitaxel plus trastuzumab after adjuvant anthracycline-based chemotherapy in patients with resected stage I disease (ClinicalTrials.gov: NCT01853748). The efficacy and safety of trastuzumab emtansine versus trastuzumab as adjuvant therapy will be assessed in the phase III KATHERINE study in patients with residual invasive disease in the breast or axillary lymph nodes following neoadjuvant trastuzumab-containing therapy (ClinicalTrials.gov: NCT01772472). An additional phase III study, KAITLIN, will evaluate the safety and efficacy of a taxane-based therapy with either trastuzumab and pertuzumab, or trastuzumab emtansine and pertuzumab in the adjuvant setting and following an anthracycline-based regimen (ClinicalTrials.gov: NCT01966471). A phase III study, KRISTINE, will evaluate the safety and efficacy of neoadjuvant trastuzumab emtansine with pertuzumab compared with chemotherapy combined with trastuzumab and pertuzumab for patients with HER2positive breast cancer (ClinicalTrials.gov: NCT02131064).

The recently developed SC formulation of trastuzumab administered once every 3 weeks for 1 year is approved globally as (neo)adjuvant treatment for HER2-positive early breast cancer. The approval was based on the results of the randomized phase III HannaH study (enHANced treatment with NeoAdjuvant Herceptin), in which the SC formulation of trastuzumab was noninferior in efficacy to intravenous (IV) administration of trastuzumab, with a similar safety profile [30]. The incidence of grade 3 or higher AEs was comparable with SC and IV administration of trastuzumab (53.5\% vs $52.3 \%$, respectively) after a median follow-up of 20 months [31]; the majority of these were hematologic toxic effects, most commonly neutropenia, leukopenia, and febrile neutropenia [30]. EFS rates at 3 years post-randomization were $76 \%$ and $73 \%$ with SC and IV administration of trastuzumab, respectively [31]. In both treatment arms, pCR was associated with improved long-term EFS: 3-year EFS rates were $19 \%$ higher in patients who achieved pCR versus those who did not [32]. The overall safety profile of SC administration of trastuzumab in HannaH was consistent with the established safety profile of IV administration of trastuzumab. Safety findings were similar with SC administration of trastuzumab in the phase II adjuvant PrefHer study (Preference for Herceptin SC or IV administration), which assessed patient preference for SC or IV administration [33, 34]. At the primary analysis, $88.9 \%(415 / 467)$ of patients preferred SC administration of trastuzumab $(p<0.0001$, two-sided test against the null hypothesis of $65 \%$ SC preference), $9.6 \%$ (45/467) preferred IV administration, and $1.5 \%$ (7/467) had no preference, with time saving $(80.3 \%)$ and less pain/discomfort/side effects (34.3\%) being the main reasons for patients' preferences [34]. Interim results from the phase III SafeHer study (Safety of assisted- and self-administered subcutaneous 
Table 2 Overview of long-term safety data from the pivotal adjuvant studies of trastuzumab

\begin{tabular}{|c|c|c|c|}
\hline \multirow[t]{2}{*}{ Grade $3 / 4$ AEs, by body system or term } & \multicolumn{3}{|l|}{ Number $(\%)$ of patients } \\
\hline & \multicolumn{3}{|l|}{ HERA 8 years' follow-up [14] } \\
\hline & 1 year trastuzumab $(n=1682)$ & 2 years trastuzumab $(n=1673)$ & Observation $(n=1744)$ \\
\hline At least one grade $3 / 4 \mathrm{AE}$ & $275(16.3)$ & $342(20.4)$ & $143(8.2)$ \\
\hline Neoplasms & $60(3.6)$ & $77(4.6)$ & $31(1.8)$ \\
\hline Infections/infestations & $37(2.2)$ & $55(3.3)$ & $12(0.7)$ \\
\hline Nervous system disorders & $14(0.8)$ & $33(2.0)$ & $12(0.7)$ \\
\hline Vascular disorders & $31(1.8)$ & $33(2.0)$ & $17(1.0)$ \\
\hline Musculoskeletal disorders & $25(1.5)$ & $28(1.7)$ & $22(1.3)$ \\
\hline General disorders/administration site conditions & $17(1.0)$ & $27(1.6)$ & $8(0.5)$ \\
\hline Gastrointestinal disorders & $17(1.0)$ & $28(1.7)$ & $6(0.3)$ \\
\hline \multirow[t]{3}{*}{ Injury/poisoning/procedural complications } & $18(1.1)$ & $23(1.4)$ & $12(0.7)$ \\
\hline & \multicolumn{3}{|l|}{ BCIRG 0065.4 years' follow-up* [12] } \\
\hline & $\mathrm{AC}-\mathrm{T}(n=1050)$ & AC-T + trastuzumab $(n=1068)$ & $\mathrm{TCH}(n=1056)$ \\
\hline Arthralgia & $34(3.2)$ & $35(3.3)$ & $15(1.4)$ \\
\hline Myalgia & $55(5.2)$ & $56(5.2)$ & $19(1.8)$ \\
\hline Hand-foot syndrome & $20(1.9)$ & $20(1.9)$ & 0 \\
\hline Stomatitis & $37(3.5)$ & $31(2.9)$ & $15(1.4)$ \\
\hline Vomiting & $65(6.2)$ & $72(6.7)$ & $37(3.5)$ \\
\hline Neutropenia & $665(63.3)$ & $764(71.5)$ & $696(65.9)$ \\
\hline Leukopenia & $544(51.8)$ & $644(60.3)$ & $509(48.2)$ \\
\hline Anemia & $25(2.4)$ & $33(3.1)$ & $61(5.8)$ \\
\hline \multirow[t]{3}{*}{ Thrombocytopenia } & $17(1.6)$ & $22(2.1)$ & $64(6.1)$ \\
\hline & \multicolumn{3}{|l|}{ NOAH 3.2 years' follow-up [13] } \\
\hline & Trastuzumab + chemotherapy $(n=115)$ & \multicolumn{2}{|l|}{ Chemotherapy alone $(n=113)$} \\
\hline Febrile neutropenia & $2(1.7)$ & \multicolumn{2}{|l|}{$2(1.8)$} \\
\hline Neutropenia & $3(2.6)$ & \multicolumn{2}{|l|}{$5(4.4)$} \\
\hline Diarrhea & $1(0.9)$ & \multicolumn{2}{|l|}{$4(3.5)$} \\
\hline Stomatitis & $1(0.9)$ & \multicolumn{2}{|l|}{$4(3.5)$} \\
\hline Pneumonia & $1(0.9)$ & \multicolumn{2}{|l|}{0} \\
\hline Arthralgia & 0 & \multicolumn{2}{|l|}{$3(2.7)$} \\
\hline Myalgia & $1(0.9)$ & \multicolumn{2}{|l|}{$1(0.9)$} \\
\hline \multirow[t]{3}{*}{ Peripheral neuropathy } & $1(0.9)$ & \multicolumn{2}{|l|}{$2(1.8)$} \\
\hline & FinHer 3 years' follow-up [19] & & \\
\hline & Docetaxel + trastuzumab $(n=54)$ & \multicolumn{2}{|l|}{ Vinorelbine + trastuzumab $(n=61)$} \\
\hline Neutropenia & $54(100)$ & \multicolumn{2}{|l|}{$34(55.7)$} \\
\hline Elevated AST & 0 & \multicolumn{2}{|l|}{$2(3.3)$} \\
\hline Stomatitis & $2(4.0)$ & \multicolumn{2}{|l|}{0} \\
\hline Allergic reaction & $3(5.8)$ & \multicolumn{2}{|l|}{0} \\
\hline Infection & $3(5.9)$ & \multicolumn{2}{|l|}{$1(1.6)$} \\
\hline Neutropenic fever & $16(29.6)$ & \multicolumn{2}{|l|}{$3(4.9)$} \\
\hline Motor neuropathy & 0 & \multicolumn{2}{|l|}{$1(1.6)$} \\
\hline Fatigue & $4(8.0)$ & \multicolumn{2}{|l|}{$3(4.9)$} \\
\hline
\end{tabular}

$A C-T$ doxorubicin and cyclophosphamide followed by docetaxel, $A E$ adverse event, $A S T$ aspartate aminotransferase, $T C H$ docetaxel and carboplatin plus trastuzumab

*Showing only AEs at $p<0.05$ for the comparison of the AC-T plus trastuzumab group versus the TCH group

Herceptin) confirmed the safety of SC administration of trastuzumab via hand-held syringe as adjuvant therapy in combination with chemotherapy for HER2-positive early breast cancer [35]. No new safety signals were identified 
and results were consistent with the known safety profile of adjuvant trastuzumab. The SC formulation of trastuzumab has now been widely adopted.

\section{Discussion}

The optimal duration of adjuvant trastuzumab treatment has been debated since the selection of the 1-year time scale based on pivotal trial results [36]. Recent data from the HERA study confirm that 2 years of trastuzumab following adjuvant chemotherapy is not more effective than 1 year [14]. Several ongoing or recently completed phase III trials address whether a shorter than 1-year course of trastuzumab might be sufficient. After 3.5 years of follow-up, the PHARE study failed to show noninferiority of 6 months versus 12 months of adjuvant administration of trastuzumab [21]. Similarly, the Hellenic Oncology Group study failed to demonstrate noninferiority for 6 months of adjuvant trastuzumab (in combination with dose-dense docetaxel following FEC) versus 12 months of treatment [37]. Results of the ongoing randomized PERSEPHONE trial comparing 6 months with 12 months of adjuvant trastuzumab (ClinicalTrials.gov: NCT00712140) and the recently completed Synergism Or Long Duration (SOLD) trial, comparing 9 weeks with 12 months of adjuvant trastuzumab (ClinicalTrials.gov: NCT00593697), are eagerly awaited [38]. At this present time, 1 year of trastuzumab treatment remains the standard of care in HER2-positive early breast cancer.

An observational study assessing the real-life use of adjuvant trastuzumab in 1245 patients with HER2-positive breast cancer reported a relapse rate of $10.9 \%$ in trastuzumab-treated patients versus $22.6 \%$ in untreated patients (median follow-up 37.4 and 62.1 months, respectively), with lower DFS in relapsed versus non-relapsed patients [39]. The relapse rate at 5 years was $17 \%$, which is in line with the BCIRG 006 study and other observational studies, but much lower than that reported in the HERA study [39].

Numerous studies, including combined results from the NSABP B-31 and NCCTG N9831 trials, have identified older age as a risk factor for trastuzumab-induced cardiotoxicity [40-44]. In addition, in a large cohort of patients with early breast cancer, older patients (age 80 years or more) had less chance of completing adjuvant trastuzumab than younger patients (66-70 years; odds ratio $0.40,95 \%$ CI $0.30-0.55$ ) [45]. Those who failed to complete the planned treatment experienced significantly higher rates of hospitalizations due to cardiac AEs than those who completed treatment $(8.1 \%$ vs $2.6 \% ; p<0.001)$. Older age was also a poor prognostic factor for RFS in patients with HER2-positive early breast cancer receiving 1 year of trastuzumab (age at least 70 years; relative risk 2.7, $95 \%$ CI 1.19-6.13; $p=0.017$ ) [46]. Future adjuvant breast cancer treatment strategies should focus on reducing cardiotoxicity and improving efficacy in elderly patients receiving trastuzumab [47].

Reducing cardiac events and preventing cardiac toxicity is a key goal in developing adjuvant treatment strategies for patients with HER2-positive breast cancer. The discovery of biomarkers or echocardiographic changes that could predict cardiac deterioration in trastuzumab-treated patients, or detect patients with the greatest chance of LVEF recovery following trastuzumab withdrawal, would be highly beneficial. A predictive role of troponins and B-type natriuretic peptides in the development of cardiac failure post-chemotherapy has been indicated in a number of publications [48]. Moreover, highly sensitive troponin $\mathrm{T}$ and cardiac diastolic function were predictive of cardiotoxicity in patients with breast cancer receiving adjuvant trastuzumab [49], while troponin I was an independent predictor of trastuzumab-induced cardiotoxicity and lack of LVEF recovery [50].

In patients with relatively small cancers, or otherwise defined as low risk of recurrence, the addition of trastuzumab may be of only limited benefit. Here the potential cardiac side effects of trastuzumab should be considered and may dissuade its use [47]. Exploratory subgroup analyses from the NCCTG N9831 study suggest that patients with high levels of stromal tumor-infiltrating lymphocytes (at least $60 \%$ of cells in the section) do not derive benefit from the addition of trastuzumab to chemotherapy, with estimated 10-year RFS rates of $90.9 \%$ with chemotherapy and $80.0 \%$ with trastuzumab plus chemotherapy $(p=0.22)$ [51]. While these data might suggest that such patients may not require trastuzumab treatment, they are hypothesis-generating and more extensive research is needed before they can be used to influence clinical decision-making. Limiting the amount of chemotherapy administered in combination with trastuzumab has also been investigated as a means of reducing the side effect burden in patients with low-risk disease. The APT trial enrolled patients with node-negative, small HER2-positive tumors (less than $3 \mathrm{~cm}$ ) to receive adjuvant paclitaxel plus trastuzumab, followed by trastuzumab monotherapy. After 3 years of follow-up, the risk of invasive recurrence was just $1.3 \%$. In this good prognosis subgroup, $6 \%$ of patients withdrew as a result of AEs; symptomatic CHF occurred in $0.5 \%$ of patients, but LVEF normalized after trastuzumab discontinuation [52]. These results demonstrate the efficacy and safety of this less intensive regimen in a lowrisk patient group often under-represented in adjuvant trastuzumab trials.

A large body of evidence is lacking for the combination of hormones and trastuzumab without chemotherapy in the adjuvant setting.

LVEF is the most commonly used marker of left ventricular systolic function and provides thresholds for intervention for patients with cardiovascular disease. The value can be derived from imaging modalities but two-dimensional (2D) transthoracic echocardiography is the most widely used as it is safe 
and cost-effective, especially for patients requiring serial scans. The main drawback is interobserver variability, but this is low with experienced operators. Three-dimensional echocardiography holds great promise for the future as the reproducibility is superior to $2 \mathrm{D}$, but the service is currently limited to a few centers. For patients with poor acoustic windows, IV contrast agents improve endocardial border definition. Studies are also focusing on the role of speckle tracking, which can be performed off-line from conventional 2D echo scans and provides information on myocardial deformation in the long, radial, and circumferential axes. This allows the detection of subclinical cardiac dysfunction before LVEF has reduced. Speckle tracking has provided insights into many disease processes but as yet has no role in the clinical setting.

Most data regarding mechanisms of cardiotoxicity due to cancer therapy have been obtained from animal models. Further studies to improve our understanding of the relevance of these pathways in humans are necessary for drug therapies to be developed. Drugs that promote increased cardiac reserve and reverse remodeling under the stress of cancer therapy are routinely used as cardioprotective measures to continue and complete therapy. These include certain beta blockers, angiotensin-converting enzyme inhibitors, and aldosterone receptor antagonists. Currently, data only exist to support use of these drugs when there is a clear reduction in LVEF rather than with subclinical left ventricular dysfunction. Omission of anthracycline therapy to avoid pre-trastuzumab damage is another strategy used in some high cardiac risk individuals.

\section{Conclusion}

Data reviewed within this paper confirm the significant survival benefit afforded by the addition of trastuzumab to chemotherapy in patients with HER2-positive early breast cancer, and the acceptable safety profile of this treatment. Long-term cardiac safety data also suggest that the incidence of cardiac AEs is maintained at a relatively low level with continued follow-up. Several ongoing adjuvant trials will define the role of newer HER2-targeted agents in early breast cancer.

Author Contributions All authors were involved in the analysis and interpretation of data included in this article, in the writing of the article, and in the decision to submit the article for publication. All authors have reviewed and approved the final version for publication.

\section{Compliance with Ethical Standards}

Funding Third-party medical writing assistance for this review paper was provided by Dr Fiona Fernando, Gardiner-Caldwell Communications and was funded by Roche Products Ltd.

Conflicts of Interest HK has received remuneration for chairing of, and participation in, advisory boards for Roche. MB has received consulting fees for occasional advisory board meetings, but outside of the submitted work. IRM reports personal fees from Roche Products UK Ltd., Pierre Fabre, Amgen, and provision of laboratory research materials from Genentech, all outside of the submitted work. LL is a former Roche employee. MP, RS, and MK declare no conflict of interest.

Open Access This article is distributed under the terms of the Creative Commons Attribution-NonCommercial 4.0 International License (http:// creativecommons.org/licenses/by-nc/4.0/), which permits any noncommercial use, distribution, and reproduction in any medium, provided you give appropriate credit to the original author(s) and the source, provide a link to the Creative Commons license, and indicate if changes were made.

\section{References}

1. Schechter AL, Stern DF, Vaidyanathan L, Decker SJ, Drebin JA, Greene MI, et al. The neu oncogene: an erb-B-related gene encoding a 185,000-Mr tumour antigen. Nature. 1984;312:513-6.

2. Coussens L, Yang-Feng TL, Liao YC, Chen E, Gray A, McGrath J, et al. Tyrosine kinase receptor with extensive homology to EGF receptor shares chromosomal location with neu oncogene. Science. 1985;230:1132-9.

3. Slamon DJ, Clark GM, Wong SG, Levin WJ, Ullrich A, McGuire WL. Human breast cancer: correlation of relapse and survival with amplification of the HER-2/neu oncogene. Science. 1987;235:177-82.

4. Shalaby MR, Shepard HM, Presta L, Rodrigues ML, Beverley PC, Feldmann M, et al. Development of humanized bispecific antibodies reactive with cytotoxic lymphocytes and tumor cells overexpressing the HER2 protooncogene. J Exp Med. 1992;175:217-25.

5. Slamon DJ, Leyland-Jones B, Shak S, Fuchs H, Paton V, Bajamonde A, et al. Use of chemotherapy plus a monoclonal antibody against HER2 for metastatic breast cancer that overexpresses HER2. N Engl J Med. 2001;344:783-92.

6. Marty M, Cognetti F, Maraninchi D, Snyder R, Mauriac L, Tubiana-Hulin M, et al. Randomized phase II trial of the efficacy and safety of trastuzumab combined with docetaxel in patients with human epidermal growth factor receptor 2-positive metastatic breast cancer administered as first-line treatment: the M77001 study group. J Clin Oncol. 2005;23:4265-74.

7. Bechmann T, Olsen DA, Jakobsen EH, Madsen JS, Brandslund I, Jylling AM, et al. Quantitative detection of HER2 protein concentration in breast cancer tissue does not increase the number of patients eligible for adjuvant HER2-targeted therapy. Oncol Rep. 2013;29:1475-82.

8. Slamon DJ, Godolphin W, Jones LA, Holt JA, Wong SG, Keith DE, et al. Studies of the HER-2/neu proto-oncogene in human breast and ovarian cancer. Science. 1989;244:707-12.

9. Piccart-Gebhart MJ, Procter M, Leyland-Jones B, Goldhirsch A, Untch M, Smith I, et al. Trastuzumab after adjuvant chemotherapy in HER2-positive breast cancer. N Engl J Med. 2005;353:1659-72.

10. Romond EH, Perez EA, Bryant J, Suman VJ, Geyer Jr CE, Davidson NE, et al. Trastuzumab plus adjuvant chemotherapy for operable HER2-positive breast cancer. N Engl J Med. 2005;353: 1673-84.

11. Smith I, Procter M, Gelber RD, Guillaume S, Feyereislova A, Dowsett M, et al. 2-year follow-up of trastuzumab after adjuvant chemotherapy in HER2-positive breast cancer: a randomised controlled trial. Lancet. 2007;369:29-36.

12. Slamon D, Eiermann W, Robert N, Pienkowski T, Martin M, Press $\mathrm{M}$, et al. Adjuvant trastuzumab in HER2-positive breast cancer. N Engl J Med. 2011;365:1273-83. 
13. Gianni L, Eiermann W, Semiglazov V, Manikhas A, Lluch A, Tjulandin S, et al. Neoadjuvant chemotherapy with trastuzumab followed by adjuvant trastuzumab versus neoadjuvant chemotherapy alone, in patients with HER2-positive locally advanced breast cancer (the NOAH trial): a randomised controlled superiority trial with a parallel HER2-negative cohort. Lancet. 2010;375:377-84.

14. Goldhirsch A, Gelber RD, Piccart-Gebhart MJ, de Azambuja E, Procter M, Suter TM, et al. 2 years versus 1 year of adjuvant trastuzumab for HER2-positive breast cancer (HERA): an openlabel, randomised controlled trial. Lancet. 2013;382:1021-8.

15. Perez EA, Romond EH, Suman VJ, Jeong JH, Davidson NE, Geyer $\mathrm{Jr}$ CE, et al. Four-year follow-up of trastuzumab plus adjuvant chemotherapy for operable human epidermal growth factor receptor 2positive breast cancer: joint analysis of data from NCCTG N9831 and NSABP B-31. J Clin Oncol. 2011;29:3366-73.

16. Perez EA, Romond EH, Suman VJ, Jeong JH, Sledge G, Geyer Jr $\mathrm{CE}$, et al. Trastuzumab plus adjuvant chemotherapy for human epidermal growth factor receptor 2-positive breast cancer: planned joint analysis of overall survival from NSABP B-31 and NCCTG N9831. J Clin Oncol. 2014;32:3744-52.

17. Perez EA, Suman VJ, Davidson NE, Gralow JR, Kaufman PA, Visscher DW, et al. Sequential versus concurrent trastuzumab in adjuvant chemotherapy for breast cancer. J Clin Oncol. 2011;29: 4491-7.

18. Gianni L, Eiermann W, Semiglazov V, Lluch A, Tjulandin S, Zambetti M, et al. Neoadjuvant and adjuvant trastuzumab in patients with HER2-positive locally advanced breast cancer (NOAH): follow-up of a randomised controlled superiority trial with a parallel HER2-negative cohort. Lancet Oncol. 2014;15:640-7.

19. Joensuu H, Kellokumpu-Lehtinen PL, Bono P, Alanko T, Kataja V, Asola R, et al. Adjuvant docetaxel or vinorelbine with or without trastuzumab for breast cancer. N Engl J Med. 2006;354:809-20.

20. Joensuu H, Bono P, Kataja V, Alanko T, Kokko R, Asola R, et al. Fluorouracil, epirubicin, and cyclophosphamide with either docetaxel or vinorelbine, with or without trastuzumab, as adjuvant treatments of breast cancer: final results of the FinHer trial. J Clin Oncol. 2009;27:5685-92

21. Pivot X, Romieu G, Debled M, Pierga JY, Kerbrat P, Bachelot T, et al. 6 months versus 12 months of adjuvant trastuzumab for patients with HER2-positive early breast cancer (PHARE): a randomised phase 3 trial. Lancet Oncol. 2013;14:741-8.

22. Spielmann M, Roché H, Delozier T, Canon JL, Romieu G, Bourgeois $\mathrm{H}$, et al. Trastuzumab for patients with axillary-nodepositive breast cancer: results of the FNCLCC-PACS 04 trial. J Clin Oncol. 2009;27:6129-34.

23. Seidman A, Hudis C, Pierri MK, Shak S, Paton V, Ashby M, et al. Cardiac dysfunction in the trastuzumab clinical trials experience. $\mathrm{J}$ Clin Oncol. 2002;20:1215-21.

24. Swain SM, Ewer MS, Cortés J, Amadori D, Miles D, Knott A, et al. Cardiac tolerability of pertuzumab plus trastuzumab plus docetaxel in patients with HER2-positive metastatic breast cancer in CLEOPATRA: a randomized, double-blind, placebo-controlled phase III study. Oncologist. 2013;18:257-64.

25. Sendur MA, Aksoy S, Yorgun H, Ozdemir N, Yilmaz FM, Yazıcı $\mathrm{O}$, et al. Comparison of the long term cardiac effects associated with 9 and 52 weeks of trastuzumab in HER2-positive early breast cancer. Curr Med Res Opin. 2015;14:1-14.

26. Yu AF, Yadav NU, Lung BY, Eaton AA, Thaler HT, Hudis CA, et al. Trastuzumab interruption and treatment-induced cardiotoxicity in early HER2-positive breast cancer. Breast Cancer Res Treat. 2015;149:489-95.

27. Herceptin prescribing information. Available at: http://www.drugs. com/pro/herceptin.html. Accessed 4 Sept 2015.

28. Jones AL, Barlow M, Barrett-Lee PJ, Canney PA, Gilmour IM, Robb SD, et al. Management of cardiac health in trastuzumabtreated patients with breast cancer: updated United Kingdom
National Cancer Research Institute recommendations for monitoring. Br J Cancer. 2009;100:684-92.

29. Curigliano G, Cardinale D, Suter T, Plataniotis G, de Azambuja E, Sandri MT, et al. Cardiovascular toxicity induced by chemotherapy, targeted agents and radiotherapy: ESMO Clinical Practice Guidelines. Ann Oncol. 2012;23 Suppl 7:vii155-66.

30. Ismael G, Hegg R, Muehlbauer S, Heinzmann D, Lum B, Kim SB, et al. Subcutaneous versus intravenous administration of (neo)adjuvant trastuzumab in patients with HER2-positive, clinical stage I-III breast cancer (HannaH study): a phase 3, open-label, multicentre, randomised trial. Lancet Oncol. 2012;13:869-78.

31. Jackisch C, Hegg R, Stroyakovskiy D, Ahn JS, Melichar B, Chen $\mathrm{SC}$, et al. Subcutaneous versus intravenous trastuzumab in early breast cancer: 2-year follow-up of HannaH. Presented at the St Gallen International Breast Cancer Conference, Vienna, Austria, 18-21 March, 2015; Poster P201.

32. Jackisch C, Hegg R, Stroyakovskiy D, Ahn JS, Melichar B, Chen SC, et al. Total pathologic complete response (tpCR) and event-free survival (EFS) with subcutaneous (SC) or intravenous (IV) trastuzumab in HER2-postive early breast cancer (EBC): the HannaH phase III study. J Clin Oncol 2015;33(Suppl.) [abstract 585].

33. Pivot X, Gligorov J, Müller V, Barrett-Lee P, Verma S, Knoop A, et al. Preference for subcutaneous or intravenous administration of trastuzumab in patients with HER2-positive early breast cancer (PrefHer): an open-label randomised study. Lancet Oncol. 2013;14:962-70.

34. Pivot X, Gligorov J, Müller V, Curigliano G, Knoop A, Verma S, et al. Patients' preferences for subcutaneous trastuzumab versus conventional intravenous infusion for the adjuvant treatment of HER2positive early breast cancer: final analysis of 488 patients in the international, randomized, two-cohort PrefHer study. Ann Oncol. 2014:25:1979-87.

35. Gligorov J, De Laurentiis M, Verrill M, Jung KH, Azim HA, Ataseven B, et al. Subcutaneous trastuzumab plus chemotherapy for early breast cancer: interim safety from SafeHer. Presented at the St Gallen International Breast Cancer Conference, Vienna, Austria, 18-21 March, 2015; Poster P103.

36. Pinto AC, Ades F, de Azambuja E, Piccart-Gebhart M. Trastuzumab for patients with HER2 positive breast cancer: delivery, duration and combination therapies. Breast. 2013;22 Suppl 2:S152-5.

37. Mavroudis D, Saloustros E, Malamos N, Kakolyris S, Boukovinas I, Papakotoulas P, et al. Six versus 12 months of adjuvant trastuzumab in combination with dose-dense chemotherapy for women with HER2-positive breast cancer: a multicenter randomized study by the Hellenic Oncology Research Group (HORG). Ann Oncol. 2015;26:1333-40.

38. Arteaga CL, Sliwkowski MX, Osborne CK, Perez EA, Puglisi F, Gianni L. Treatment of HER2-positive breast cancer: current status and future perspectives. Nat Rev Clin Oncol. 2011;9:16-32.

39. Mustacchi G, Puglisi F, Molino AM, Crivellari D, Ghiotto C, Ferro A, et al. Observational study on adjuvant trastuzumab in HER2-positive early breast cancer patients. Future Oncol. 2015;11:1493-500.

40. Russell SD, Blackwell KL, Lawrence J, Pippen Jr JE, Roe MT, Wood F, et al. Independent adjudication of symptomatic heart failure with the use of doxorubicin and cyclophosphamide followed by trastuzumab adjuvant therapy: a combined review of cardiac data from the National Surgical Adjuvant Breast and Bowel Project B31 and the North Central Cancer Treatment Group N9831 clinical trials. J Clin Oncol. 2010;28:3416-21.

41. Bonifazi M, Franchi M, Rossi M, Moja L, Zambelli A, Zambon A, et al. Trastuzumab-related cardiotoxicity in early breast cancer: a cohort study. Oncologist. 2013;18:795-801.

42. Chen J, Long JB, Hurria A, Owusu C, Steingart RM, Gross CP. Incidence of heart failure or cardiomyopathy after adjuvant trastuzumab therapy for breast cancer. J Am Coll Cardiol. 2012;60:2504-12. 
43. Tarantini L, Gori S, Faggiano P, Pulignano G, Simoncini E, Tuccia $\mathrm{F}$, et al. Adjuvant trastuzumab cardiotoxicity in patients over 60 years of age with early breast cancer: a multicenter cohort analysis. Ann Oncol. 2012;23:3058-63.

44. Romond EH, Jeong JH, Rastogi P, Swain SM, Geyer Jr CE, Ewer MS, et al. Seven-year follow-up assessment of cardiac function in NSABP B-31, a randomized trial comparing doxorubicin and cyclophosphamide followed by paclitaxel (ACP) with ACP plus trastuzumab as adjuvant therapy for patients with node-positive, human epidermal growth factor receptor 2-positive breast cancer. J Clin Oncol. 2012;30:3792-9.

45. Vaz-Luis I, Keating NL, Lin NU, Lii H, Winer EP, Freedman RA. Duration and toxicity of adjuvant trastuzumab in older patients with early-stage breast cancer: a population-based study. J Clin Oncol. 2014;32:927-34.

46. Tonyali O, Coskun U, Sener N, Inanc M, Akman T, Ulas A, et al. Prognostic factors for recurrence-free survival in patients with HER2-positive early-stage breast cancer treated with adjuvant trastuzumab. Onkologie. 2013;36:554-8.

47. Puglisi F, Fontanella C, Amoroso V, Bianchi GV, Bisagni G, Fontana A, et al. Current challenges in HER2-postive breast cancer. Crit Rev Oncol Hematol. 2016;98:211-21.
48. Stachowiak P, Kornacewicz-Jach Z, Safranow K. Prognostic role of troponin and natriuretic peptides as biomarkers for deterioration of left ventricular ejection fraction after chemotherapy. Arch Med Sci. 2014;10:1007-18.

49. Katsurada K, Ichida M, Sakuragi M, Takehara M, Hozumi Y, Kario $\mathrm{K}$. High-sensitivity troponin $\mathrm{T}$ as a marker to predict cardiotoxicity in breast cancer patients with adjuvant trastuzumab therapy. Springerplus. 2014;3:620.

50. Cardinale D, Colombo A, Torrisi R, Sandri MT, Civelli M, Salvatici $\mathrm{M}$, et al. Trastuzumab-induced cardiotoxicity: clinical and prognostic implications of troponin I evaluation. J Clin Oncol. 2010;28: 3910-6.

51. Perez EA, Ballman KV, Anderson KA, Thompson EA, Badve SS, Bailey H, et al. Stromal tumor-infiltrating lymphocytes (S-TILs): in the alliance N9831 trial S-TILs are associated with chemotherapy benefit but not associated with trastuzumab benefit. Presented at the San Antonio Breast Cancer Symposium, San Antonio, Texas, 9-13 December, 2014; Abstract S1-06.

52. Tolaney SM, Barry WT, Dang CT, Yardley DA, Moy B, Marcom PK, et al. Adjuvant paclitaxel and trastuzumab for node-negative, HER2-positive breast cancer. N Engl J Med. 2015;372:134-41. 